\title{
Efecto de dos tasas de riego y apoyo de sombra sobre la tasa de supervivencia y crecimiento de un arbusto nativo En Peligro de la Región de Coquimbo
}

\author{
Effects of two irrigation rates and shade on survival and plant \\ growth in an endangered native shrub of Coquimbo Region \\ Marta Vargas $^{1}$, Mariano Rodriguez-Malebrán ${ }^{1}$, Carlos Navarrete ${ }^{2}$, Cristian Ibáñez ${ }^{1 *}$
}

\section{RESUMEN}

El objetivo de este trabajo fue evaluar el efecto de dos tasas de riego y apoyo de sombra sobre la tasa de supervivencia y crecimiento de Myrcianthes coquimbensis, un arbusto en Peligro de Extinción, endémico del secano costero de la Región de Coquimbo. Las evaluaciones se realizaron durante 24 meses. Durante los tres primeros meses, las plantas fueron regadas con 12 o $24 \mathrm{~L} / \mathrm{mes}$, después del cual el riego se suspendió hasta el final del ensayo. Los resultados obtenidos mostraron que la aplicación de un riego de $12 \mathrm{~L} /$ mes durante los tres primeros meses aseguró una supervivencia inicial de las plantas cercanas al 100\%, independiente de si tenían o no sombra. Desde el momento que el riego fue suspendido (inicio del cuarto mes post-plantación), el efecto de la sombra se hizo importante. Así, mientras las plantas sin sombra redujeron su porcentaje de supervivencia al 20\%, con un incremento temporal al $30 \%$ debido a lluvias invernales, y un descenso durante el segundo año en torno a $15 \%$, las plantas con sombra mantuvieron valores de supervivencia que bordearon el 50\% durante el primer año y en torno al 30\% de supervivencia al final del segundo año. Los resultados muestran que cualquier estrategia de forestación que se intente en esta especie nativa debería ir acompañada de un riego de establecimiento mínimo de $12 \mathrm{~L} / \mathrm{mes}$ y el apoyo de sombra. Este estudio sirve como herramienta de apoyo para conservación in situ de este amenazado arbusto endémico de Chile.

Palabras claves: Myrtacea, forestación, zonas áridas, conservación in situ.

\section{ABSTRACT}

The aim of this work was to evaluate the effect of two irrigation rates and shade on survival and plant growth parameters of Myrcianthes coquimbensis, an endangered and endemic shrub of the coastal dry area of the Coquimbo Region. Evaluations were performed for 24 months. During the first three months, the plants were watered with 12 or 24 L/month, after which the irrigation was suspended until the end of the study. The results showed that the application of an irrigation of $12 \mathrm{~L} /$ month during the first three months ensured an initial survival of plants close to $100 \%$, regardless of whether or not they had shade. During this initial period, the use of shade only allowed to observe significant differences in the growth of new shoots. From the moment the irrigation was suspended (beginning of the fourth month post-afforestation), the effect of the shade became important. Thus, while shade-less plants reduced their survival rate by around $20 \%$, with a temporary increase to $30 \%$ after winter rains and drop at about $15 \%$ by the second year, shady plants maintained survival values near to 50\% during first year and 30\% at the end of the study. The results showed that any afforestation strategy attempted in this native shrub should be accompanied by a minimum establishment irrigation rate of $12 \mathrm{~L}$ month and permanent shadow support. This study is a contribution for the in situ conservation of this valuable and unique shrub of Chile.

Keywords: Myrtacea, afforestation, arid zones, in situ conservation.

\section{Introducción}

Lucumillo (Myrcianthes coquimbensis (Barnéoud) Landrum et Grifo, familia Myrtaceae), es un arbusto nativo endémico de la Región de Coquimbo y único representante de este género en Chile (Landrum y Grifo, 1988). Su rango de distribución comprende una zona muy acotada en la

\footnotetext{
$1 \quad$ Universidad de La Serena, Departamento de Biología. La Serena. Chile.

2 Universidad de La Serena, Departamento de Matemáticas. La Serena. Chile.

* Autor por correspondencia: cibanez@userena.cl.
}

Fecha de Recepción: 20 Abril, 2017.

Fecha de Aceptación: 12 Octubre, 2017.

DOI: 
costa de la provincia del Elqui, desde Barrancones $\left(29^{\circ} 25^{\prime} \mathrm{S}-71^{\circ} 79^{\prime} \mathrm{O}\right)$ hasta Las Tacas $30^{\circ} 04^{\prime} \mathrm{S}$ $71^{\circ} 22^{\prime}$ O) (García-Guzmán et al., 2012). El lugar está inserto en una zona árida caracterizada por las escasas precipitaciones y una alta radiación solar (Santibáñez et al., 2008). En abril de 2008 y siguiendo los criterios de la Unión Internacional para la Conservación de la Naturaleza (UICN), el Ministerio de Medio Ambiente de Chile declaró oficialmente a $M$. coquimbensis como una especie "En Peligro", asignándole el código EN B1ab (iii) + 2ab (iii)). Este código hace referencia a que la especie se encuentra En Peligro (EN), tiene una distribución muy restringida $\left(132 \mathrm{~km}^{2}, \mathrm{~B} 1\right)$, su hábitat está severamente fragmentado (B1a), se ha producido una disminución del hábitat (B1b(iii)), que su área de ocupación es menos de $500 \mathrm{Km}^{2}$ (B2), el cual se encuentra severamente fragmentado (B2a) y la calidad de este ha disminuido continuamente (B20(iii) (Riedermann et al., 2006). Basado en estos antecedentes, la conservación in situ y ex situ de esta especie de alto valor ecológico se ha vuelto esencial como miras a su preservación. Para la conservación ex situ, ya se cuenta con protocolos para propagarla vía sexual y asexualmente (Saldías y Velozo, 2014; Loayza et al., 2014). Sin embargo, para la conservación in situ, no se cuenta aún con un plan que defina áreas de conservación o áreas de manejo dentro de su rango de distribución. En este sentido, generar experiencias para determinar, entre otros factores, cuáles son los requerimientos del establecimiento de plantas juveniles de la especie en su rango de distribución y cómo estas responden al establecimiento asistido e interacción con su medio ambiente, son aspectos necesarios de conocer para contribuir de manera concreta a la recuperación y conservación in situ de M. coquimbensis. Esto adquiere aun mayor relevancia al constatar la poca regeneración natural que muestra esta especie nativa a lo largo de su acotado rango de distribución (García-Guzmán et al., 2012).

Para establecer especies nativas en zonas áridas, la disponibilidad de agua es sin lugar a dudas el factor más limitante para el éxito del establecimiento (Cerda et al., 2011). Sin embargo, Santelices et al. (2011) destacan que el uso de cubiertas que protejan a las plantas es un factor que también es relevante, sobre todo cuando se foresta en zonas con radiación elevada o viento constante, que son justamente dos condiciones que prevalecen en las zonas costeras donde habita esta especie.

Basado en lo anterior, se propuso como objetivo de esta investigación: 1) evaluar el efecto de dos regímenes de riego y el factor sombra a través del uso de malla raschel sobre la supervivencia y crecimiento de plantas de $M$. coquimbensis, 2) evaluar la supervivencia después de un periodo estival sin riego, con miras a entender cuál de los dos factores (riego o sombra artificial) tiene incidencia mayor en el establecimiento, y 3) observar si las precipitaciones ocurridas a lo largo de un periodo de evaluación de dos años tienen algún efecto en la supervivencia o rescate de individuos plantados.

\section{Métodos}

\section{Características del sitio de estudio}

El sitio elegido correspondió a una terraza marina plio-cuaternaria (Paskoff, 1999) ubicada en la Región de Coquimbo, Provincia de Elqui, sector Bahía El Arrayán $\left(29^{\circ} 43^{\prime} \mathrm{S}, 71^{\circ} 1^{\prime} 9^{\prime} \mathrm{O}\right.$, 50 m.s.n.m), el cual se encuentra al centro de su distribución natural de la especie (GarcíaGuzmán et al., 2012) (Figura 1A). La Bahía El Arrayán ha sido declarada por el Libro Rojo de la Región de Coquimbo (Squeo et al., 2001) como un área de alto interés ecológico e importante de conservar debido a la gran diversidad de especies y endemismos allí identificados. Cabe señalar que todo el sector Bahía El Arrayán se caracteriza por una creciente fragmentación de su hábitat debido fundamentalmente a la construcción de viviendas y habilitación de caminos. El terreno elegido para la forestación se encontraba debidamente cercado para protegerlo de la fauna autóctona y exótica, y su distancia del borde costero no superaba los $200 \mathrm{~m}$, lo cual aseguraba una fuerte influencia del clima marino.

Según la clasificación basada en el criterio de las Naciones Unidas para la tipificación de regímenes de humedad, $M$. coquimbensis se desarrolla en una zona árida (Santibáñez et al., 2008). La precipitación en dicha zona ocurre de manera discreta (pulsos de lluvia con características torrenciales) e infrecuente, concentrándose en los meses de invierno y difícilmente supera los $100 \mathrm{~mm}$ año ${ }^{-1}$ (Gutiérrez, 2008; Santibáñez et al., 2008). Las excepciones se dan en años de ocurrencia intensa del fenómeno de El Niño-Oscilación del 

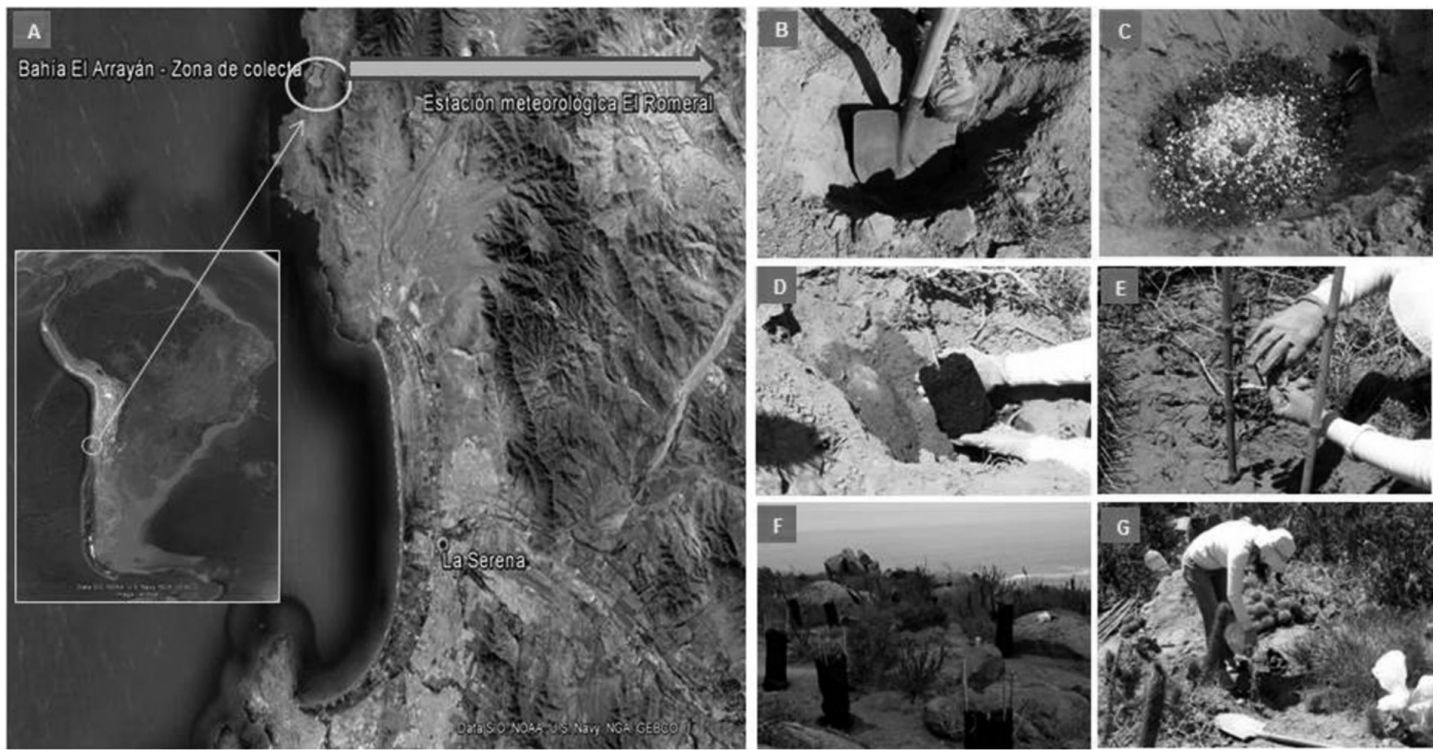

Figura 1. Establecimiento de plantas en el sector de estudio. A) Sector de estudio, ampliada desde la imagen de Sudamérica y que muestra su ubicación respecto a La Serena y Estación meteorológica El Romeral. B) Hoyadura. C) Humedecimiento de la hoyadura, aplicación de tierra de hojas y fertilizante base. D) Plantación. E) Identificación de réplicas y tratamientos. F) Vista general de disposición de plantas, donde se aprecia al fondo la cercanía del Océano Pacífico. G) Riego de establecimiento. Imágenes satelitales mostradas en A) obtenidas desde Google Earth el día 5 de diciembre, 2016.

Sur (ENOS), pudiendo entonces las precipitaciones superar los $100 \mathrm{~mm}_{\text {año }}{ }^{-1}$ (Soto et al., 2015). El periodo seco se extiende por 8 a 11 meses y el índice de humedad invernal varía de 0,5 a 1 , lo que evidencia una corta estación húmeda de uno o dos meses. El régimen térmico acumula anualmente entre 1600 y 1700 días-grados (Santibáñez et al., 2008). En la Tabla 1 se muestra el registro de precipitaciones mensuales ocurrido en los años 2014, 2015 y 2016 en la Estación meteorológica El Romeral (294' $13^{\circ}$ "S, $71^{\circ} 15^{\prime} 21^{\prime \prime}$ O, 162 m.s.n.m), ubicada $\sim 10 \mathrm{~km}$ de distancia del lugar de estudio (CEAZAMET, 2016), y que corresponde a la Estación Meteorológica más cercana al área de estudio. Su ubicación se indica en la Figura 1A. Otros datos meteorológicos registrados en la misma Estación como la temperatura de aire y suelo, humedad radiación PAR y viento para el mismo periodo de años son mostrados en Anexo Tabla 1.

\section{Establecimiento de plantas}

Un total de 120 plantas fueron establecidas en una superficie aproximada de $2000 \mathrm{~m}^{2}$ (Figura 1B a G). Para ello, se procedió a cavar una hoyadura de $25 \mathrm{~cm}$ de profundidad, eligiéndose al azar un lugar que estuviese alejado de las rocas. Luego, se humedeció la hoyadura con agua, se aplicó tierra de hojas y fertilizante base, se mezclaron manualmente y se procedió a la plantación. Con cuidado de no dañar las raíces, las plantas

Tabla 1. Precipitaciones mensuales observadas en la zona de estudio desde los 2014 a 2016. Los subíndices indican pulsos de lluvia ocurridos durante dichos meses.

\begin{tabular}{lccc}
\hline \multicolumn{4}{c}{ Precipitaciones $(\mathrm{mm})$ mensuales } \\
\hline Mes/Año & 2014 & 2015 & 2016 \\
\hline Enero & 0,1 & 0 & 0,5 \\
Febrero & 0 & 0 & 0,1 \\
Marzo & 0,4 & $7,5^{1}$ & 0,1 \\
Abril & 0 & 0 & 2,2 \\
Mayo & 0,1 & 0 & 2,4 \\
Junio & 24,5 & 0 & 2,2 \\
Julio & 0,1 & $35^{2,3}$ & $15,7^{7}$ \\
Agosto & 0,1 & $44^{4}$ & 0,1 \\
Septiembre & 17,2 & $10,5^{5}$ & 0,6 \\
Octubre & 0 & 28,96 & $4,2^{8}$ \\
Noviembre & 0 & 0 & 0 \\
Diciembre & 0,7 & 0 & 0 \\
\hline Total & 43,2 & 125,9 & 28,1 \\
\hline
\end{tabular}

Pulsos de precipitaciones: ${ }^{1}=7,2 \mathrm{~mm}(25$ marzo $){ }^{2}=24 \mathrm{~mm}$ (12 julio); ${ }^{3}=10,8 \mathrm{~mm}$ (13 julio); ${ }^{4}=38,4 \mathrm{~mm}(8$ agosto $) ;{ }^{5}=$ $10,3 \mathrm{~mm}$ ( 24 septiembre $) ;{ }^{6}=28,5 \mathrm{~mm}(14$ octubre $) ;{ }^{7}=12 \mathrm{~mm}$ (25 julio); ${ }^{8}=4.2 \mathrm{~mm}$ (16 octubre) (CEAZAMET 2016). 
de $M$. coquimbensis fueron dispuestas en las hoyaduras y luego cubiertas con tierra del mismo lugar. Posteriormente, cada planta fue etiquetada para su identificación de réplica y tratamiento correspondiente, finalizando luego con un riego inicial de establecimiento equivalente a $6 \mathrm{~L}$ de agua por planta. La Figura 1 muestra gráficamente el procedimiento de plantación. Las plantas utilizadas en este estudio fueron cultivadas en el vivero del Departamento de Biología de la Universidad de La Serena y al momento de la plantación tenían entre 1 a 1,5 años de edad, con un promedio de diámetro de cuello a los $55 \mathrm{~cm}$ de 2,3 $\mathrm{mm} \pm 2,1$, y una altura promedio de $51,35 \mathrm{~cm} \pm 17,9$.

\section{Diseño experimental y mediciones}

Se utilizó un diseño factorial $2 \times 2$ donde el primer factor fueron dos condiciones de riego mensual: $12 \mathrm{~L} / \mathrm{mes}$ (Riego I, RI) y $24 \mathrm{~L} / \mathrm{mes}$ (Riego II, RII). El segundo factor correspondió a dos condiciones de sombra: Con Sombra (generada con el uso de una malla raschel a $80 \%$ de densidad, y con intensidad lumínica promedio en su interior de $522,86 \pm 252.41 \mu \mathrm{mol} \mathrm{m}^{-2} \mathrm{~s}^{-1}$ ), y Sin Sombra (exposición directa al sol, sin uso de malla raschel e intensidad lumínica promedio de 3045,86 \pm $1026.18 \mu \mathrm{mol} \mathrm{m}^{-2} \mathrm{~s}^{-1}$ ) (Figura 1). Las intensidades lumínicas se hicieron con luminómetro LI-COR ${ }^{\circledR}$ Biosciences, modelo Li-250A. Por cada tratamiento se utilizaron 30 individuos dispuestos al azar sobre el terreno. La evaluación de parámetros se realizó a $\operatorname{los} 3,6,7,12$ y 24 meses post-plantación.

Durante los primeros tres meses se mantuvieron las dos condiciones de riego y las dos condiciones de sombra con el fin de evaluar el comportamiento inicial de $M$. coquimbensis al establecimiento. En concreto, la evaluación de esta etapa se desarrolló desde la primera semana de octubre de 2014 hasta la primera semana de enero de 2015 , registrándose al final de este periodo el porcentaje de supervivencia. En esta primera etapa se evaluó la altura total de la planta $(\mathrm{cm})$ para determinar si hubo o no hubo crecimiento (CR), y utilizando un análisis de regresión logística se evaluó la significancia estadística de este parámetro. Los resultados fueron expresados como razón de oportunidades relativas (odds ratio, OR) con $95 \%$ de intervalo de confianza. El OR se define como un cociente entre dos oportunidades (odds). En este caso, un OR correspondió a la oportunidad que plantas crecieran con riego I y sin malla dividido por la oportunidad que plantas crecieran con riego II y con malla. Se contabilizó la cantidad de brotes nuevos (BN) que fue analizado mediante un análisis de varianza de dos vías con posterior prueba de Tukey, mientras que la longitud de cada brote (cm) (LB) se analizó con la prueba de Kruskal-Wallis, y la comparación de las medianas se realizó con la prueba de Dunn. Para evaluar el riesgo de mortalidad se ajustó un modelo Probit Multivariado Bayesiano, donde las respuestas fueron los vectores $\boldsymbol{Y}_{i}=\left(Y_{i 1}, \cdots, Y_{i 5}\right)$, para $i=1, \ldots, 116$ donde los valores $\boldsymbol{Y}_{\mathrm{ij}}, \mathrm{j}=1, \ldots, 5$ corresponden a los estados de Vida (1) o Muerte (0) para la planta $i$ en los tiempos 3, 6, 7,12 y 24 meses. El modelo considera cada vector $\boldsymbol{Y}_{i}$ como reflejo de un vector latente $\boldsymbol{Z}_{i}$ de distribución Normal Multivariada de media $\beta \mathbf{X}_{i}$ (definida de forma análoga a un modelo ANOVA con interacción) y matriz de correlación estimada a través de su descomposición de Cholesky. La relación entre $\boldsymbol{Y}$ y $\boldsymbol{Z}$ está dada por $Y_{i j}=I\left(Z_{i j}\right.$ $>0$ ), donde $I(\cdot)$ es una función indicadora. La distribución a priori para los parámetros $\beta$ fue definida Normal de media 0 y desviación estándar 5. La estimación para la sobrevivencia promedio de las plantas para cada tratamiento se basó en las medidas a posteriori (M.A.P.) $\mathrm{P}\left(Z_{i j}>0 \mid \boldsymbol{Y}\right.$, $\beta)$ obtenidas mediante métodos Markov Chain Monte Carlo (MCMC). Los análisis estadísticos se realizaron con el programa $\mathrm{R}$ versión 3.0.3. ( $\mathrm{R}$ Development Core Team, 2016) y Stan Version 2.10 (Stan Development Team Version, 2015).

Una vez terminado el tercer mes postplantación (primera semana de enero de 2015), las dos condiciones de riego fueron suspendidas y el porcentaje de supervivencia fue evaluado al final del mes 6, 7, 9, 12 y 24 post-plantación. Durante todo este periodo, se mantuvieron las condiciones de sombra, las plantas no fueron fertilizadas y el agua que recibieron correspondió exclusivamente a aquella que precipitó de manera natural sobre el lugar de estudio (Tabla 1).

\section{Resultados y Discusión}

En general, se observó una buena supervivencia en todos los tratamientos de cultivo a los tres meses post-plantación, encontrándose solo una planta muerta (tratamiento RI con malla), equivalente al 3,6\% del total de plantas de este tratamiento. En el resto de los tratamientos hubo un 100\% 
de supervivencia (Figura 2). Entre los factores que podrían haber influido positivamente en los resultados de establecimiento inicial destaca la edad de las plantas utilizadas. Así, Becerra et al. (2013) obtuvieron un supervivencia del $77.8 \%$ en plantas de mayor edad en comparación al $62.6 \%$ obtenido con plantas más jóvenes en las especies esclerófilas Maytenus boaria, Quillaja saponaria, Lithrea caustica, Schinus latifolius, Schinus polygamus y Kageneckia oblonga. Así, el desarrollo y edad de las plantas que se destinan a una plantación podría ser un atributo importante de calidad en $M$. coquimbensis cuando se quiere establecer en su rango de distribución. Duryea (1985) define calidad como "la capacidad para alcanzar unas expectativas de supervivencia y crecimiento en una estación particular".

Para el crecimiento (Tabla 2), se encontró que plantas de M. coquimbensis con Riego II (24 L/

Tabla 2. Probabilidad de supervivencia entre tratamientos (Riego y sombra) y crecimiento de plantas de M. coquimbensis después de tres meses post-plantación.

OR = Oportunidad Relativa; Límite inferior y Límite superior indican el valor mínimo y máximo que adquiere el intervalo de confianza (95\%) del OR; P = Probabilidad de observar un evento por simple azar, dada la hipótesis nula.

\begin{tabular}{lrcrr}
\hline Tratamiento & OR & $\begin{array}{c}\text { Límite } \\
\text { inferior }\end{array}$ & $\begin{array}{c}\text { Límite } \\
\text { superior }\end{array}$ & Valor-P \\
\hline (Intercepto) & $-1,14$ & 0,37 & $-3,12$ & $<0,01$ \\
Riego II & 1,35 & 0,40 & 3,33 & $<0,01$ \\
Con malla & 0,76 & 0,40 & 1,89 & 0,06 \\
\hline
\end{tabular}

mes) tuvieron una razón de oportunidad (OR) de $1,35(0,40 ; 3,33)(95 \%$ IC), lo que indica que estas plantas crecieron 1,35 veces más que el grupo control (Riego I sin sombra). Cuando las plantas no tuvieron sombra, la razón de oportunidades (OR) fue $0,76(0,40 ; 1,89)$, el cual no fue significativo $(P$ $=0.06$ ). Esto indicaría que la presencia de sombra en esta especie no influyó en el crecimiento a los tres meses de plantación, ni tampoco en el número de brotes. En cambio, el riego sí que influyó significativamente a este parámetro $(\mathrm{F}=$ $11,03, P<0,01, \mathrm{n}=30$ ). Plantas que recibieron $24 \mathrm{~L} / \mathrm{mes}$ generaron en promedio 5,26 brotes por planta mientras que aquellas regadas con $12 \mathrm{~L} /$ mes tuvieron en promedio 3,23 brotes por planta (Figura 3). En relación al promedio de longitud de brotes por planta se tuvo diferencia significativa tanto para el factor riego $\left(\mathrm{X}^{2}=8,32, P<0,01\right.$, $\mathrm{df}=1, \mathrm{n}=60)$ como para el factor sombra $\left(\mathrm{X}^{2}=\right.$ 22,64, $P<0,01, \mathrm{df}=1, \mathrm{n}=60$ ). Así, mayor riego (24 L/planta) generó en promedio una longitud de $1,85 \mathrm{~cm}$ en comparación a los $1,25 \mathrm{~cm}$ que tuvieron plantas regadas con $12 \mathrm{~L} / \mathrm{mes}$, lo que significa que la tasa de $24 \mathrm{~L} / \mathrm{mes}$ si influyó en que los brotes fuera un tercio más largos en un periodo de tres meses. En cuanto al efecto de la sombra, su uso generó brotes con un crecimiento promedio de $1,95 \mathrm{~cm}$ mientras que sin sombra, este crecimiento promedio alcanzó $1,0 \mathrm{~cm}$. Por tanto, el efecto de la presencia de sombra generó aproximadamente el doble de crecimiento. Al comparar los cuatro tratamientos se obtuvo diferencias significativas $\left(\mathrm{X}^{2}=31,22, \mathrm{P}<0,01, \mathrm{df}=3, \mathrm{n}=30\right)$, presentándose

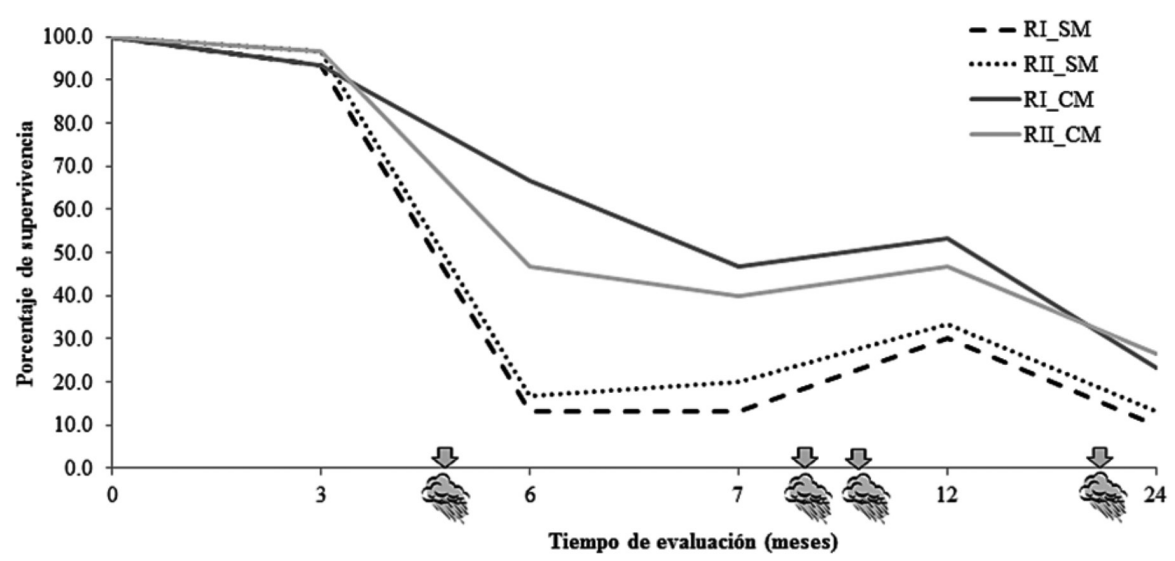

Figura 2. Evaluación de la supervivencia (\%) de plantas de Myrcianthes coquimbensis durante 24 meses según dos tipos de riegos ( $\mathrm{RI}=12 \mathrm{~L} / \mathrm{mes}$, RII= $24 \mathrm{~L} / \mathrm{mes}$ ) y uso $(\mathrm{CM})$ o no uso $(\mathrm{SM})$ de sombra. Las flechas y nubes indican pulsos de precipitaciones registradas en el periodo, los cuales son detallados en la Tabla 1. 


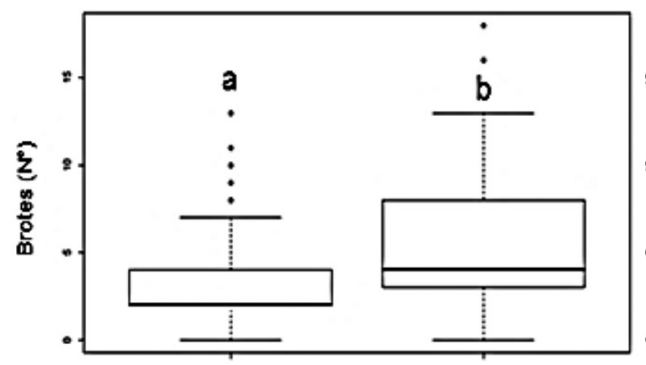

।

\|

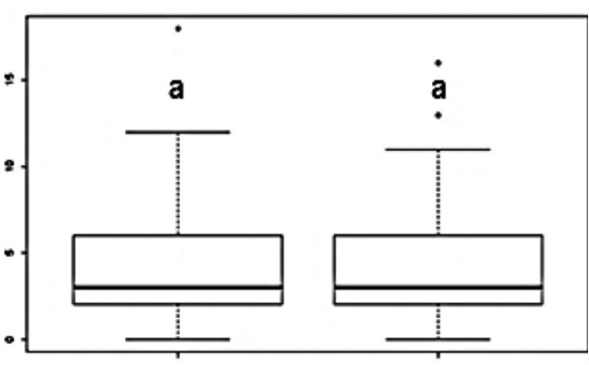

sm

\section{Riego}

Figura 3. Cantidad de brotes nuevos según tasa de riego ( $\mathrm{RI}=12 \mathrm{~L} / \mathrm{mes}$, RII $=24 \mathrm{~L} / \mathrm{mes})$ y uso $(\mathrm{CM})$ o no uso (SM) de sombra a los tres meses de iniciado el establecimiento.

un mayor crecimiento en brotes de plantas bajo el tratamiento de Riego II (24 L/mes) con sombra. Éstas crecieron en promedio $2,525 \mathrm{~cm}$ que equivale aproximadamente a un $150 \%$ más en comparación a los otros tres tratamientos (Figura 4). Al respecto, Mora y Meneses (2003) confirman la necesidad de la aplicación de agua para el crecimiento y producción incluso en especies de escaso requerimiento hídrico que son establecidas en una zona árida, como fue comprobado con Acacia saligna en la comuna de Ovalle. Por otra parte, si bien no se sabe con exactitud cuál es la tasa mínima de riego que requiere $M$. coquimbensis, los resultados demuestran que la adición de $12 \mathrm{~L} / \mathrm{mes}$ de agua permitió su establecimiento en la época de primavera. A su vez, Donoso et al. (2006) demostraron que ante una mayor restricción hídrica, Quillaja saponaria no presentó un mecanismo claro de ajuste al déficit hídrico pero redujo su biomasa foliar en un 55\%.

Al analizar la supervivencia al sexto mes post-plantación, y a tres meses de haber cesado los riegos, en todos los ensayos evaluados el porcentaje de supervivencia bajó considerablemente aunque la mortalidad de plantas fue menor en aquellas que tenían apoyo de sombra (Figura 2). Así, mientras las plantas sin sombra con riegos iniciales de 12 $\mathrm{L} / \mathrm{mes}$ y $24 \mathrm{~L} / \mathrm{mes}$ bajaron sus porcentajes de supervivencia a $13,3 \%$ y $16,7 \%$ respectivamente, las plantas que tenían sombra tuvieron porcentajes de supervivencia de $66,7 \%$ y $46,7 \%$, respectivamente (Figura 2). Durante el transcurso de esta etapa del estudio (enero a abril 2015) ocurrió una inusual lluvia de verano tardía de 7,2 mm (25/marzo/2015, Tabla 1) que no generó cambios inmediatos en la tasa de supervivencia. Sin embargo, al cabo de dos meses de transcurrida la lluvia sorpresivamente algunas plantas del tratamiento de $24 \mathrm{~L} / \mathrm{mes}$ sin malla comenzaron a rebrotar mientras que la curva descendente de supervivencia del tratamiento de $12 \mathrm{~L} / \mathrm{mes}$ sin sombra se detuvo y pareció estabilizarse (Figura 2). En los tratamientos con sombra, en cambio, el pulso de agua no pareció

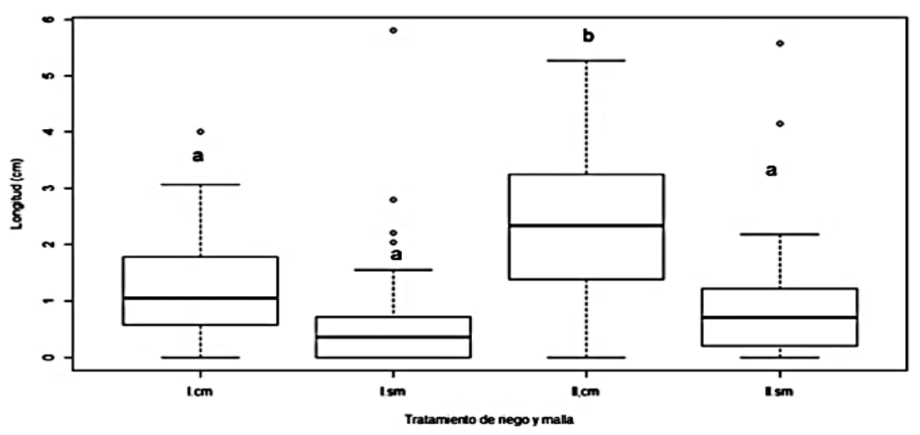

Figura 4. Crecimiento ( $\mathrm{cm}$ ) de brotes nuevos en plantas de $M$. coquimbensis según tasa de riego $(\mathrm{RI}=12 \mathrm{~L} / \mathrm{mes}, \mathrm{RII}=24 \mathrm{~L} / \mathrm{mes})$ y uso $(\mathrm{CM})$ o no uso $(\mathrm{SM})$ de sombra a los tres meses post plantación. 
influir la tendencia negativa que mostraba la curva de supervivencia pues esta continuó descendiendo (Figura 2).

Al final del mes 12 post-plantación, las plantas del tratamiento $12 \mathrm{~L} / \mathrm{mes} \sin$ sombra (Riego I) y que habían sido las más afectadas por la suspensión del riego duplicaron el porcentaje de supervivencia de $13,3 \%$ a $30 \%$ (Figura 2). Por su parte, las plantas del tratamiento de Riego II ( $24 \mathrm{~L} / \mathrm{mes})$ sin sombra aumentaron el porcentaje de supervivencia de $16,7 \%$ a $33,3 \%$. En cuanto a los tratamientos con sombra, si bien no se evidenció una recuperación de plantas como la observada en los tratamientos sin sombra, si fue posible observar que en ambos tratamientos (Riego I y II), la tendencia descendente de la curva de supervivencia observadas al termino del sexto y séptimo mes fueron revertidas durante este periodo de evaluación. Así, mientras el tratamiento de Riego I con sombra terminó el periodo de 12 meses post-plantación con 53,3\% de supervivientes, el tratamiento de Riego II con sombra lo hizo con un 46,7\%. La recuperación de las plantas se asocia con las intensas lluvias que ocurrieron durante este periodo. Como se muestra en la Tabla 1, entre junio y la primera semana de octubre de 2015 hubieron cuatro pulsos de agua, siendo los más intensos los del 12 julio $(24 \mathrm{~mm} /$ día) y 8 de agosto $(38,4 \mathrm{~mm} /$ día), seguidos por los del 13 julio (10,8 mm/día) y 24 de septiembre (10,3 mm/día). Al finalizar el año 2015, se registró una precipitación anual igual a 125,9 mm.

$\mathrm{Al}$ analizar la supervivencia de las plantas al final del segundo año de evaluación (octubre de 2016), y comparado al observado al final del primer año, en todos los tratamientos hubo una pérdida aproximada del $50 \%$ de individuos. Así, el porcentaje de supervivencia para los tratamientos de Riego I y II sin sombra bajaron de $30 \%$ y $33 \%$ a $10 \%$ y $13,3 \%$, respectivamente. En los mismos tratamientos de riego pero con apoyo de sombra, los porcentajes de supervivencia bajaron de 53,3\% y $46,7 \%$ a $23,3 \%$ y $27 \%$, respectivamente (Figura 2). Esta disminución importante de individuos vivos podría explicarse por la escasa precipitación que hubo durante el año 2016 y en que solo se registraron dos pulsos de agua (versus seis del año anterior) y sus intensidades fueron muy inferiores a los pulsos del año anterior. Específicamente, el $25 \mathrm{de}$ julio precipitaron $12 \mathrm{~mm} /$ día y el 16 de octubre precipitaron 4,2 $\mathrm{mm} /$ día (Tabla 1), totalizando una precipitación anual para el año 2016 de tan solo
28,1 mm (Tabla 1). Si bien es cierto que la escasa precipitación pudo gatillar la pérdida de individuos, es importante señalar que los resultados mostraron que aquellas plantas que tuvieron apoyo de sombra mostraron una pérdida menor. Esto quiere decir, que en caso que una plantación de esta especie no pueda ser regada, o reciba una baja cantidad de precipitaciones, entonces el uso de sombra tendría un efecto positivo sobre su establecimiento. Al respecto, Hernández y Vita (2004) reportaron altos porcentajes de supervivencia en el Parque Nacional Bosque de Fray Jorge para Aextoxicon punctatum (Olivillo), Myrceugenia correifolia (Petrillo) y Drimys winteri (canelo), con 97\%, 83\% y $74 \%$, respectivamente, en que durante el primer año la aplicación de una tasa de riego semanal fue de 5 litros, seguido por una aplicación de $5 \mathrm{~L}$ cada 15 días en el segundo año. Considerando este aspecto, pareciera que el riego de mantención en $M$. coquimbensis debería extenderse por un periodo más prolongado que tres meses si se desea favorecer la supervivencia de plantas hasta un segundo año de plantación, mas considerando lo errático que es el régimen pluvial del territorio en que se encuentra distribuida esta especie.

De acuerdo a los resultados del modelo Probit Multivariado Bayesiano (Tabla 3), las mayores diferencias entre tratamientos (con nivel de credibilidad sobre 95\%) fueron, por una parte, la mayor supervivencia de plantas que tuvieron apoyo de sombra para el nivel de riego de $12 \mathrm{~L} /$ mes y que se evidenciaron a partir de los seis meses post-plantación. Bajo esta misma condición de riego y después de 2 años de realizada la plantación, el porcentaje de supervivientes que tenían apoyo de sombra triplicó al de plantas que nunca lo tuvieron. Por otro lado, para $24 \mathrm{~L} / \mathrm{mes}$ la inclusión de sombra incidió en el porcentaje de supervivientes también a partir de los 6 meses, sin embargo, al final de los 2 años post-plantación estas diferencias se acortaron entre las plantas con y sin apoyo de sombra. En vista de los resultados anteriores podemos inferir que luego de un periodo estival sin apoyo de riego artificial (equivalente a 6 meses post-plantación en nuestro estudio), el factor sombra fue preponderante en la supervivencia de $M$. coquimbensis, afectando su ausencia en mayor medida a aquellas plantas que había recibido una tasa de riego de $12 \mathrm{~L} / \mathrm{mes}$ en comparación a aquellas plantas que recibieron $24 \mathrm{~L} / \mathrm{mes}$. En este último caso, la ausencia de 
Tabla 3. Porcentaje de supervivencia (Intervalo de credibilidad 95\%)

para los tratamientos de riego y sombra a lo largo de 2 años de estudio.

\begin{tabular}{cccccc}
\hline \multicolumn{3}{c}{ Porcentaje de supervivencia (I.C. 95\%) } \\
\cline { 2 - 3 } Tiempo (Meses) & \multicolumn{2}{c}{$12 \mathrm{~L} / \mathrm{mes}$} & & \multicolumn{2}{c}{$24 \mathrm{~L} / \mathrm{mes}$} \\
\cline { 2 - 3 } & Con Malla & Sin Malla & & Con Malla & Sin Malla \\
\hline 3 & $0,97(0,85 ; 1,00)$ & $0,93(0,80 ; 0,99)$ & & $0,96(0,82 ; 1,00)$ & $0,97(0,85 ; 1,00)$ \\
6 & $0,67(0,52 ; 0,84)$ & $0,13(0,04 ; 0,27)$ & & $0,42(0,24 ; 0,62)$ & $0,17(0,05 ; 0,30)$ \\
7 & $0,47(0,28 ; 0,64)$ & $0,13(0,04 ; 0,28)$ & & $0,35(0,19 ; 0,54)$ & $0,20(0,06 ; 0,33)$ \\
24 & $0,53(0,36 ; 0,72)$ & $0,30(0,17 ; 0,49)$ & & $0,42(0,26 ; 0,63)$ & $0,33(0,19 ; 0,54)$ \\
& $0,27(0,12 ; 0,42)$ & $0,10(0,03 ; 0,24)$ & & $0,31(0,16 ; 0,50)$ & $0,20(0,07 ; 0,35)$ \\
\hline
\end{tabular}

sombra pero mayor disponibilidad de agua permitió mantener una mayor supervivencia final. Entre los factores que podrían explicar estas diferencias en la supervivencia en favor del uso de sombra, Puerta-Piñero et al. (2007) destacan la importancia que tiene el empleo de cubiertas que protejan a las plantas de ciertas especies, por ejemplo, de una radiación solar demasiado elevada o contra fuertes vientos, pues ambos son factores que aceleran la tasa de evapotranspiración de las plantas (Valipour, 2015) y con ello causantes de estrés y mortalidad de plántulas. Acorde con los resultados, efectivamente el uso de sombra pudo haber reducido la exposición de las plantas tanto a la alta radiación solar como a la incesante brisa marina que ocurre en la zona de estudio, y cuyo registro de radiación PAR alcanzó un máximo de $2838 \mu \mathrm{mol} \mathrm{m}^{-2} \mathrm{seg}^{-1}$ en diciembre de $2015 \mathrm{y}$ una velocidad máxima del viento de $19,6 \mathrm{~m} \mathrm{~s}^{-1}$ en agosto de 2015.

El uso de sombra podría también estar ejerciendo un efecto de atrapanieblas. En este sentido, experiencias realizadas en los años 2011 y 2012 en Punta Gruesa, (20 km al sur de Iquique), en que se evaluó el efecto de atrapanieblas en la restauración y recuperación de Eulychnia iquiquensis, se concluyó que el uso de atrapanieblas tuvo un efecto positivo en el crecimiento de las plantas tanto en la altura como en la anchura de los tallos. Adicionalmente, el atrapanieblas contribuyó en el desarrollo de nuevos brotes y en el cambio en el colorido de la epidermis de esta cactácea, pasando de un verde amarillento a un verde intenso (Pinto, 2014). En el caso de $M$. coquimbensis, inspecciones visuales realizadas al término del estudio demostraron que las plantas que tenían malla raschel poseían hojas más verdes y con aspecto más saludable que aquellas que nunca tuvieron la protección de las mallas.

Finalmente, los pulsos de agua recibidos por esta especie parecen actuar como un factor de retardo de la mortalidad de las plantas, como ha sido sugerido por Alameda y Villar (2009) en especies leñosas de ecosistemas mediterráneos. A diferencia de lo que ocurre en plantas anuales (Armesto et al., 1993), los pulsos de agua en $M$. coquimbensis parecieron efectivos en la medida que estos mantuvieron una periodicidad en el tiempo. Así, las intensas lluvias ocurridas durante el año 2015, que superaron con creces las precipitaciones observadas en la zona y que no se sostuvieron en el año 2016, trajo consigo que la tasa de supervivencia volviera a los valores registrados antes del inicio de las lluvias de 2015. Este hecho indica que si a esta especie no se le apoya con riego en años con baja cantidad de precipitaciones, probablemente la expectativa de supervivencia no será muy alentadora. Por otro lado, este hecho refuerza lo indicado por Gutiérrez (2008), quien señala la importancia que tiene el fenómeno ENOS en la recuperación y mantención de la vegetación árida de Chile.

En resumen, ante el desafío que supone establecer M. coquimbensis en su hábitat árido es esencial considerar riego periódico (al menos cada dos semanas) durante los primeros meses postplantación y repetir los riegos en caso de inviernos con baja cantidad de precipitación. Así mismo, sería aconsejable un uso de sombra para evitar la deshidratación prematura de las plantas, puesto que ambos son factores que afectan y contribuyen a la supervivencia, crecimiento y producción de biomasa en esta especie nativa, endémica de Chile y En Peligro de Extinción. 


\section{Conclusiones}

El establecimiento de plantas de M. coquimbensis en el secano costero de la Región de Coquimbo es posible, siempre y cuando se apoye de riego y de sombra. En una etapa inicial, el riego es fundamental puesto que la aplicación de un riego de establecimiento de $6 \mathrm{~L} /$ planta y la mantención del riego con una tasa de $12 \mathrm{~L} / \mathrm{mes}$ asegura una alta supervivencia, crecimiento y producción de biomasa en las plantas luego de tres meses post-plantación. Ante la restricción de agua, $M$. coquimbensis reduce su tasa de supervivencia, no obstante, dicha disminución es menor en plantas que tienen apoyo de sombra. La ocurrencia de pulsos de lluvia intensos contribuye a mantener con vida a las plantas establecidas, demostrando así la importancia que tienen estos pulsos hídricos en la recuperación y mantención de plantas de zonas áridas.

\section{Agradecimientos}

Agradecemos a la Dirección de Investigación y Desarrollo de la Universidad de La Serena, proyecto DIDULS Regular 90/2014. Marta Vargas agradece al Concurso DIDULS Apoyo Tesis de Postgrado PT14121. Agradecemos a Claus Westphal por su apoyo durante la etapa de establecimiento.

\section{Literatura Citada}

Alameda, D.; Villar, R.

2009. Moderate soil compaction: Implications on growth and architecture in seedlings of 17 woody plant species. Soil \& Tillage Research, 103: 325-331.

Armesto, J.; Vidiella, P.; Gutiérrez, J.

1993. Plant communities of the fog-free coastal desert of Chile: plant strategies in a fluctuating environment. Revista Chilena de Historia Natural, 66: 271-282.

Becerra, P.; Cruz, G.; Ríos, S.; Castelli, G.

2013. Importance of irrigation and plant size in the establishment success of different native species in a degraded ecosystem of central Chile. Bosque, 34 (1): 103-111.

Ceazamet

2016. Laboratorio de Meteorología del Centro de Estudios Avanzados en Zonas Áridas. Estación El Romeral. URL: http:// www.ceazamet.cl/index.php?p_cod=ceazamet\&pag=mod_ sensor\&e_cod $=5 \& s \_c o d=25 \&$ intervalo $=$ mes $\&$ fecha $=2015-01-01$ Consultado: 7 marzo 2017.

Cerda, J.; Medina, R.; Jalil, F.

2011. Manual de Plantaciones en Zonas Áridas. Departamento Forestal-CONAF. Región de Coquimbo, Chile. 114 pp.

Donoso, S.; Peña, K.; Pacheco, C.; Luna, G.; Aguirre, A. 2006. Physiological and growth response in Quillaja saponaria and Cryptocarya alba plants under restricted water conditions. Bosque, 32 (2): 187-195.

Duryea, M.

1985. Evaluating seedling quality: Importance to reforestation. In Duryea M.L.(Ed) Evaluating seedling quality: Principles, procedures, and predictive abilities of mayor test. Forest Research Laboratory. Oregon State University, Corvallis. US. pp. 1-4.

García-Guzmán, P.; Loayza, A.; Carvajal, D.; Leteliera, L.; Squeo. F.

2012. The ecology, distribution and conservation status of Myrcianthes coquimbensis: a globally endangered endemic shrub of the Chilean Coastal Desert. Plant Ecology \& Diversity, 5 (2): 197-204.

Gutiérrez, J.

2008. El desierto florido en la Región de Atacama. Capítulo 15. En: Squeo, F.; Arancio, G.; Gutiérrez, J. (eds.), Libro
Rojo de la Flora Nativa y de los Sitios Prioritarios para su Conservación: Región de Atacama. Ediciones Universidad de La Serena. La Serena, Chile. pp. 285-291.

Hernández, I.; Vita, A.

2004. Reforestación para la expansión de los bosquetes de Olivillo. Capítulo 18. En: Squeo, F.; Gutiérrez, J.; Hernández, I. (eds.). Historia Natural del Parque Nacional Bosque Fray Jorge. Ediciones Universidad de La Serena. La Serena, Chile. pp. 307-319.

Landrum, L.; Grifo, F.

1988. Myrcianthes (Myrtaceae) in Chile. Brittonia, 40 (3): 290-293.

Loayza, A.; Carvajal, D.; García-Guzmán, P.; Squeo, F.

2014. Lucumillo: Una planta en peligro de extinción. En: SEREMIA MA. El lucumillo: Un patrimonio de la Región de Coquimbo. Ministerio de Medio Ambiente, Región de Coquimbo, Chile. Pp. 6-11.

Mora, F.; Meneses, R.

2003. Efecto del riego sobre el crecimiento y producción forrajera de Acacia saligna (Labill.) H. Wendl. en la zona árida de Chile. Cerne, 9 (2): 255-260.

Paskoff, R.

1999. Contribuciones recientes al conocimiento del Cuaternario marino del centro y norte de Chile. Revista de Geografía Norte Grande, 26: 43-50.

Pinto, R.

2014. Eulychnia iquiquensis (Schumann) Britton \& Rose: uso de atrapanieblas para la restauración y recuperación de poblaciones en peligro en el extremo norte de Chile. Chloris Chilensis. 17 (2). [on line]. Disponible en: http:// www.chlorischile.cl Consultado: 21 enero 2017

Puerta-Piñero, C.; Gómez, J.; Valladares, F.

2007. Irradiance and oak seedling survival and growth in a heterogeneous environment. Forest Ecology and Management, 242: 462-469.

$\mathrm{R}$ Core Team.

2016. R: A language and environment for statistical computing. R Foundation for Statistical Computing, Vienna, Austria. URL https://www.R-project.org/. Consultado: 21 enero 2017. 
Riedemann, P.; Aldunate, G.; Teillier, S.

2006. Flora nativa de valor ornamental Chile, zona Norte: Identificación y propagación. 1st ed. Santiago, Chile: Editorial Flora de Chile. Santiago, Chile. 440 pp.

Saldías, G.; Velozo, J.

2014. Estudio de la propagación de Myrcianthes coquimbensis (Barnéoud) Landrum et Grifo por semillas y esquejes. Gayana Bot., 71 (1): 17-23.

Santelices, R.; Cerrillo, R.; Drake, F.; Mena, C.

2011. Efecto de la cobertura y de la fertilización en el desarrollo de plantas de Nothofagus alessandrii cultivadas en contenedor. Bosque, 32 (1): 85-88.

Santibáñez, F.; Roa, P.; Santibáñez, P.

2008. El medio Físico. Capítulo I. En: Rovira, J.; Ugalde, J. y Stutzin, M. (eds.). Biodiversidad de Chile. Patrimonios y desafíos. Comisión nacional del medio ambiente. Segunda edición. Santiago, Chile. pp. 21-45.
Soto, M.; Märker, M.; Castro, C.P.; Rodolfi, G.

2015. Análisis integrado de las condiciones de amenaza natural en el medio ambiente costero semiárido de Chile. La Serena, Coquimbo. Boletín de la Asociación de Geógrafos Españoles, 67: 213-231.

Squeo, F., Arancio, G.; Gutiérrez, J.

2001. Capítulo 11. Libro rojo de la flora nativa y de los sitios prioritarios para su conservación: Región de Coquimbo. Ediciones Universidad de La Serena. La Serena, Chile. pp. 2-17.

Stan Development Team.

2015. Stan: A C ${ }^{++}$Library for Probability and Sampling, Version 2.10.0. Disponible en: http://mc-stan.org/. Consultado: 21 enero 2017.

Valipour, M.

2015. Importance of solar radiation, temperature, relative humidity, and wind speed for calculation of reference evapotranspiration. Archives of Agronomy and Soil Science, 61 (2): 239-255.

\section{Anexo}

Tabla Anexo 1. Registro meteorológico para Humedad, Temperatura, Temperatura del suelo, Máxima radiación PAR y Máxima velocidad del viento en la E.M El Romeral desde 2014 a 2016.

\begin{tabular}{|c|c|c|c|c|c|c|c|c|c|c|c|c|c|c|c|}
\hline \multicolumn{4}{|c|}{$\begin{array}{l}\text { Humedad } \\
(\%)\end{array}$} & \multicolumn{3}{|c|}{$\begin{array}{l}\text { Temperatura } \\
\left({ }^{\circ} \mathrm{C}\right)\end{array}$} & \multicolumn{3}{|c|}{$\begin{array}{l}\text { Temperatura del suelo } \\
\qquad(0.1 \mathrm{~m})\end{array}$} & \multicolumn{3}{|c|}{$\begin{array}{l}\text { Max Radiación PAR } \\
\left(0 \mathrm{~m} ; \mu \mathrm{mol} \mathrm{m} \mathrm{m}^{-2} \mathrm{~s}^{-1}\right)\end{array}$} & \multicolumn{3}{|c|}{$\begin{array}{l}\text { Max Velocidad del } \\
\text { viento }\left(2 \mathrm{~m} ; \mathrm{m} \mathrm{s}^{-1}\right)\end{array}$} \\
\hline Mes/Año & 2014 & 2015 & 2016 & 2014 & 2015 & 2016 & 2014 & 2015 & 2016 & 2014 & 2015 & 2016 & 2014 & 2015 & 2016 \\
\hline Enero & 70,04 & 76,24 & 70,29 & 18,53 & 16,7 & 19,12 & 28,42 & 25,69 & 29,53 & 2462 & 2454 & 2791 & 8,94 & 10,37 & 15,88 \\
\hline Febrero & 71,41 & 73,64 & 73,86 & 17,46 & 17,46 & 18,43 & 26,12 & 25,84 & 28,23 & 2207 & 2387 & 2494 & 11,24 & 10,39 & 11,45 \\
\hline Marzo & 77,31 & 74,3 & 76,91 & 15,1 & 17,98 & 16,6 & 22,86 & 25,33 & 25,11 & 2244 & 2083 & 2603 & 10,55 & 10,05 & 10,35 \\
\hline Abril & 74,92 & 76,42 & 75,67 & 13,72 & 15,6 & 15,41 & 19,52 & 21,34 & 21,35 & 1830 & 2002 & 2117 & 10,56 & 11,39 & 15,7 \\
\hline Mayo & 74,52 & 76,79 & 77 & 12,77 & 13,26 & 14,22 & 17,06 & 17,72 & 18,32 & 1563 & 1545 & 1842 & 11,6 & 13,33 & 12,82 \\
\hline Junio & 71,89 & 67,85 & 74,33 & 11,42 & 11,89 & 11,88 & 13,32 & 15,22 & 15,24 & 1416 & 1316 & 1557 & 10,82 & 7,35 & 11,58 \\
\hline Julio & 71,36 & 77,97 & 78,6 & 11,6 & 11,17 & 11,8 & 14,2 & 13,59 & 15,19 & 1420 & 1564 & 1612 & 11,7 & 11,25 & 9,13 \\
\hline Agosto & 73,52 & 76,98 & 74,36 & 11,94 & 13,03 & 12,18 & 16,41 & 14,95 & 17,16 & 1870 & 1890 & 2041 & 10,49 & 19,62 & 11,58 \\
\hline Septiembre & 75,55 & 77,54 & 74,06 & 12,63 & 13,4 & 12,18 & 17,64 & 18,74 & 19,37 & 2073 & 2258 & 2196 & 9,64 & 11,11 & 12,98 \\
\hline Octubre & 75,62 & 73,06 & 70,00 & 13,93 & 14,22 & 14,33 & 21,41 & 21,36 & 22,48 & 2332 & 2420 & 2554 & 11,68 & 12,72 & 13,39 \\
\hline Noviembre & 73,73 & 71,45 & 70,12 & 14,09 & 15,03 & 15,72 & 23,39 & 24,02 & 25,41 & 2414 & 2792 & 2725 & 10,19 & 11,88 & 10,04 \\
\hline Diciembre & 72,65 & 74,24 & 68,33 & 15,39 & 16,24 & 17,32 & 25,51 & 27,18 & 28,05 & 2728 & 2838 & 2707 & 11,7 & 12,17 & 12,66 \\
\hline Total & 73,54 & 74,71 & 73,63 & 14,05 & 14,67 & 14,93 & 20,49 & 20,92 & 22,12 & 2047 & 2129 & 2270 & 10,76 & 11,80 & 12,30 \\
\hline
\end{tabular}

